\title{
Exploring the Role of the Nucleus Accumbens in Adaptive Behavior Using Concurrent Intracranial and Extracranial Electrophysiological Recordings in Humans
}

\author{
DDadine Eijsker, ${ }^{1,2}$ Guido van Wingen, ${ }^{1,2}$ Ruud Smolders, ${ }^{1,2}$ Dirk J. A. Smit, ${ }^{1,2}$ and Damiaan Denys ${ }^{1,2}$
}

https://doi.org/10.1523/ENEURO.0105-20.2020

${ }^{1}$ Department of Psychiatry, Amsterdam Neuroscience, Amsterdam UMC, University of Amsterdam, Amsterdam, 1105 AZ, The Netherlands and ${ }^{2}$ Amsterdam Brain and Cognition, University of Amsterdam, Amsterdam, 1001 NK, The Netherlands

\begin{abstract}
Recent human electrophysiological evidence implicated $\theta$-band communication between the nucleus accumbens (NAc) and frontal and parietal cortex in cognitive flexibility. Since the NAc is connected with the motor system, we tested whether phase and amplitude-based NAc-cortical connectivity and power modulation likewise underlie flexibility in motor action control. We combined concurrently recorded intracranial and extracranial electroencephalograms from seven psychiatric patients implanted with deep brain stimulation (DBS) electrodes who performed a stop signal task (SST). Inhibition success, as opposed to failure, was associated with greater prestimulus information flow from right NAc to medial frontal cortex through phase coupling of $\theta$ oscillations. Inhibition failure evoked $\theta$ power increases in the left NAc and medial frontal cortex, whereas parieto-occipital cortex showed an $\alpha$ power decrease. We conclude that NAc-to-frontal $\theta$ connectivity, possibly facilitating processing of task-relevant information, and $\alpha$ and $\theta$ power modulations, possibly reflecting posterror engagement of cognitive control, contribute to adaptive behavior pertaining motor control.
\end{abstract}

Key words: cortico-striatal connectivity; electroencephalography; intracranial EEG; spectral power; stop signal task; $\theta$ oscillations

\section{Significance Statement}

Combining unique intracranial recordings from human nucleus accumbens (NAc) and concurrently recorded electroencephalographic (EEG) data, we complement previous research on the involvement of NAc-cortical $\theta$-band communication in adaptive behavior by showing that prestimulus $\theta$ phase synchronization likely drives this process.

Received March 18, 2020; accepted September 10, 2020; First published November 9, 2020.

The authors declare no competing financial interests.

Author contributions: G.v.W., R.S., and D.D. designed research; R.S. performed research; N.E. and D.J.A.S. analyzed data; N.E., G.v.W., R.S., D.J. A.S., and D.D. wrote the paper.

Acknowledgements: We thank all patients for their participation.

Correspondence should be addressed to Nadine Eijsker at nadine. eijsker@gmail.com.

https://doi.org/10.1523/ENEURO.0105-20.2020

Copyright ( 2020 Eijsker et al.

This is an open-access article distributed under the terms of the Creative Commons Attribution 4.0 International license, which permits unrestricted use, distribution and reproduction in any medium provided that the original work is properly attributed.

\section{Introduction}

The nucleus accumbens (NAc) has a well-established role in reward processing and reinforcement learning (Cohen et al., 2009a, 2012; Lega et al., 2011; Patel et al., 2012). However, in recent years, it has additionally been implicated in cognitive flexibility (Floresco et al., 2006; van Schouwenburg et al., 2010; Yawata et al., 2012; Horschig et al., 2015). Specifically, interplay between the NAc and prefrontal cortex seems important for flexibility. The prefrontal cortex is thought to exert cognitive control by strategy development and active maintenance of goal-relevant representations (Miller and Cohen, 2001) and projects directly to the NAc, whereas the NAc seems to actively gate such task- 
Table 1: Subject information and SST performance

\begin{tabular}{|c|c|c|c|c|c|c|c|c|c|}
\hline ID & Sex & Age & Diagnosis & SSRT & Mean SSD & $\begin{array}{l}\text { Mean RT } \\
\text { correct go }\end{array}$ & $\begin{array}{l}\text { Mean RT } \\
\text { failed } \\
\text { inhibition }\end{array}$ & $\begin{array}{l}\text { \% Successful } \\
\text { inhibition }\end{array}$ & $\begin{array}{l}\% \text { Incorrect } \\
\text { go }\end{array}$ \\
\hline Patient 1 & $\mathrm{~F}$ & 40 & OCD & 238 & 653 & 925 & 840 & 58 & 1 \\
\hline Patient 3 & $\mathrm{~F}$ & 32 & OCD & 305 & 138 & 433 & 412 & 44 & 0 \\
\hline Patient 4 & $\mathrm{~F}$ & 31 & OCD & 233 & 587 & 853 & 744 & 60 & 2 \\
\hline Patient 5 & $\mathrm{~F}$ & 63 & MDD & 308 & 271 & 625 & 541 & 53 & 13 \\
\hline $\begin{array}{l}\text { Summary } \\
\text { mean (SD) }\end{array}$ & $5 \mathrm{~F} / 2 \mathrm{M}$ & 40 (14.3) & & 255 (47.9) & 405 (228.4) & 679 (194.9) & 594 (176.7) & $52 \%(5.6)$ & $2 \%(4.6)$ \\
\hline
\end{tabular}

SSRT, stop signal reaction time; SSD, stop signal delay; RT, reaction time; F/M, female/male; OCD, obsessive-compulsive disorder; MDD, major depressive disorder; SUD, substance use disorder; SD, standard deviation.

relevant information (van Schouwenburg et al., 2010; Horschig et al., 2015) and indirectly projects back to frontal and parietal cortex via the globus pallidus, subthalamic nucleus and the medial dorsal nucleus of the thalamus (Alexander et al., 1986; Haber et al., 1995; Haber and Knutson, 2010). This idea was previously corroborated and extended using human intracranial electrophysiological data to show that the NAc increased $\theta$-band communication to the neocortex, primarily frontal cortex and additionally parieto-occipital cortex, on processing of visual stimuli in a task requiring an attentional switch (Horschig et al., 2015). Moreover, these regions communicated with the NAc in the $\alpha$-band during anticipation of visual processing.

Considering its connections, the NAc is seen as a functional link between the limbic and motor systems (Mogenson et al., 1980), yet the previously employed tasks only probed cognitive flexibility. Electrophysiological evidence for the involvement of the subthalamic nucleus, part of the motor system via which the NAc projects to the cortex, has already been found on a stop signal task (SST; Ray et al., 2012), a frequently adopted paradigm to study the ability to inhibit an ongoing motor response in the face of changing demands (response inhibition). Based on the NAc's involvement in cognitive flexibility and its connections to the motor system, we tested whether the NAc is likewise involved in flexibility that pertains motor action directly. We likewise adopted the SST, which requires balancing speed (rapid response to a go-signal initiating action) and accuracy (successful inhibition of an ongoing response following a stop signal). The task is theoretically grounded in the horse-race model (Logan and Cowan, 1984), which posits that response inhibition depends on the relative finishing times of independent and competing go and stop processes. However, it may not be that simple; studies have found stimulus detection and action selection and execution to be influenced by both proactive and reactive control processes, with responses often being slowed to balance accuracy and speed (Bissett and Logan, 2011).

NAc-targeted deep brain stimulation (DBS) in compulsive and depressed patients offers the unique opportunity of recording intracranial electroencephalography (iEEG) from the human NAc and surrounding area. Since bidirectional cortico-striatal communication seems essential for optimization of flexible behavior, we combined intracranial and surface EEG recordings from psychiatric patients to investigate amplitude and phase-based cortico-striatal communication and power modulation during SST performance. If the role of the NAc in behavioral flexibility is similar to that in cognitive flexibility, we expect (1) $\alpha$-band connectivity, specifically information flow from the cortex toward the NAc, during anticipation of stimulus presentation; followed by (2) $\theta$-band connectivity, specifically information flow from the NAc toward the cortex, during stimulus processing.

\section{Materials and Methods}

\section{Participants}

Ten treatment-refractory psychiatric patients were recruited from the Academic Medical Center outpatient clinic. Two participants were excluded based on performance; one successfully inhibited on over $90 \%$ of stop trials, whereas the other completely lacked successful stop trials, resulting in too few trials in the remaining condition to analyze. Another participant displayed extreme amounts of $\beta$-band oscillations because of brain tumor removal. Of the remaining seven participants (aged 22-63 years; five females and two males), four were diagnosed with obsessive-compulsive disorder (OCD; one wth comorbid obsessive-compulsive personality disorder), two patients with major depressive disorder, and one patient with cocaine and opiate addiction (Table 1). All participants were right-handed and took their standard medication, with the exception of selective serotonin reuptake inhibitors. Medications included Euthyrox $50 \mathrm{mcg} / \mathrm{d}$, Omeprazole $40 \mathrm{mg} / \mathrm{d}$, Simvastatin $20 \mathrm{mg}$, Triazolam $100 \mathrm{mg} / \mathrm{d}$, Suboxone $4 \mathrm{mg} / \mathrm{d}$, Flucloxacilline $1000 \mathrm{mg} /$ four daily, Nifedipine $40 \mathrm{mg} / \mathrm{d}$, Selokeen $50 \mathrm{mg} / \mathrm{d}$, Omeprazole $20 \mathrm{mg} / \mathrm{d}$, Melatonin $5 \mathrm{mg} / \mathrm{d}$, Promethazine $25 \mathrm{mg} / \mathrm{d}$, Lorazepam $2,5 \mathrm{mg} / \mathrm{d}$, Seroquel $300 \mathrm{mg} / \mathrm{d}$, Parnate $30 \mathrm{mg} / \mathrm{two}$ daily, Domperidon $10 \mathrm{mg} / \mathrm{d}$, and Movicolon and Paracetamol where necessary.

The local Medical Ethical Committee of the Academic Medical Center approved the experiment and all participants provided written informed consent before the experiment.

\section{Stop signal task}

Stimulus presentation

Stimuli were presented using Presentation (version 14.5; Neurobehavioral Systems) on a 15.4-inch laptop 
(HP 6730b) screen, placed $\sim 60 \mathrm{~cm}$ from the participants, at a resolution of 1024 by 768 pixels and with a refresh rate of $60 \mathrm{~Hz}$.

\section{Task properties}

The SST consists of two types of trials. In go trials, a green arrow (go stimulus) pointing either to the left or right signals participants to press the corresponding, left or right, shift button on a keyboard as fast as possible using their left and right index fingers, respectively. In stop trials, the arrow changes color from green to red (stop stimulus) after a variable delay; the stop signal delay (SSD). This signals participants to cancel their motor response to the go stimulus. Participants were instructed to respond to go signals as fast as possible, while simultaneously minimizing inhibition failures, and that these two criteria were equally important.

Our task consisted of three blocks of 100 trials each, of which $70 \%$ go trials and $30 \%$ stop trials. The intertrial interval, during which a fixation cross was presented, varied randomly between $1750,1875,2000,2125$, and $2250 \mathrm{~ms}$, with each interval presented equally often. Go stimuli were presented until response or a stop stimulus appeared, with a maximum of $1200 \mathrm{~ms}$. The SSD started at $300 \mathrm{~ms}$ and was increased and decreased with $50 \mathrm{~ms}$ after every successful and failed inhibition trial, respectively, for the left and right-hand side independently. This double staircase procedure thus increased inhibition difficulty, by increasing the amount of time between go and stop stimuli, after successful inhibition and vice versa after failed inhibition, which steers toward generating approximately equal numbers of successful and failed inhibition trials. The SSD was not reset between blocks.

\section{Data acquisition}

\section{iEEG recordings}

Patients were bilaterally implanted with deep brain electrodes (Medtronic model 3387) in the NAc between 2010 and 2012. Stereotactical placement of the electrodes was performed as previously described by van den Munckhof et al. (2013), which included planning based on T1weighted magnetic resonance images, online measurement over the electrodes to inform when the gray matter target was reached, and subsequent confirmation with a postoperative CT scan. Each electrode contained four contact points, each being $1.5 \mathrm{~mm}$ in length and separated by $0.5 \mathrm{~mm}$. The most ventral contact point was positioned in the NAc core, with the other contact points extending into the ventral part of the anterior limb of the internal capsule. Our sample performed the SST on day 4 after surgery for implantation of the deep brain electrodes, except for patient 7 , who was tested on the fifth day after surgery. Patients would later undergo surgery for implantation of the stimulator.

\section{EEG recordings}

EEG was recorded at a sampling rate of $512 \mathrm{~Hz}$ using a 64-channel recording system with shielded $\mathrm{Ag} / \mathrm{AgCl}$ electrodes (Advanced Neuro Technology B.V.) following the international 10-10 system. Of the 64 channels, eight (four per electrode) were used for the iEEG and four for collecting horizontal and vertical eye-movement. No signals were recorded from the areas covered by postsurgery bandages. Data were online common average referenced.

\section{Data analysis}

\section{Behavioral performance}

Performance measures were calculated over all trials available before artifact rejection. Stop signal reaction time (RT) was calculated using the quantile method (Verbruggen and Logan, 2009), which is less susceptible to violations of assumptions underlying the horse-race model than other methods (Band et al., 2003; Verbruggen and Logan, 2009). Per individual, this included calculating the quantile RT (QRT), which is the correct go trial RT (sorted ascendingly) corresponding to the quantile of the proportion of failed stop trials, and subsequently subtracting the mean SSD.

\section{Preprocessing}

Data were preprocessed using the EEGLAB-toolbox (version 14.1.1; Delorme and Makeig, 2004) in MATLAB (version R2014b; The MathWorks). Signals with a SD below $10 \%$ of the median or $>10$ times the median, for EEG and iEEG signals separately, were considered flatlines and too noisy, respectively, and rejected. Then, signals were re-referenced to the average of the respective signal type, i.e., EEG and iEEG. For iEEG, this was done separately for the left and right hemispheres. Signals were bandpass filtered (FIR filter with Hamming window) between 1 and $47 \mathrm{~Hz}$ and down-sampled to $256 \mathrm{~Hz}$. The latter was done to improve performance of the EEGLABplugin Automatic Artefact Rejection (Gomez-Herrero et al., 2006), using canonical correlation analysis algorithms for blind source separation, which was adopted for automatic removal of muscular artefacts in the EEG signals only. Subsequently, EEG signals were visually inspected and channels considered too noisy were rejected, after which they again were re-referenced to the mean of remaining channels. Then, we extracted epochs from -1000 to $1200 \mathrm{~ms}$ relative to go stimulus onset, which we corrected for baseline activity and visually inspected to reject epochs containing artifacts (all except eye blinks). We used principal component analysis to reduce data dimensionality to 45 components (with the exception of 42 components for one patient with $<45$ EEG channels at this point) and exclude minor components. We then ran an independent component analysis on the remaining signals and rejected components containing eye blinks and other noise. We then extracted two subsets of data; from -550 to $550 \mathrm{~ms}$ relative to stop signal onset (stop trials only) and to motor response (correct go and failed inhibition trials). For the latter, we excluded trials that contained multiple motor responses and randomly selected an equal number of go trials to keep the number of trials equal between conditions, as there was less failed inhibition than go trials available per participant. We did not do this for the stop trials, because of their limited numbers. Based on previous literature (Cohen et al., 2009b; Horschig et al., 2015), we filtered all signals (using a FIR filter 


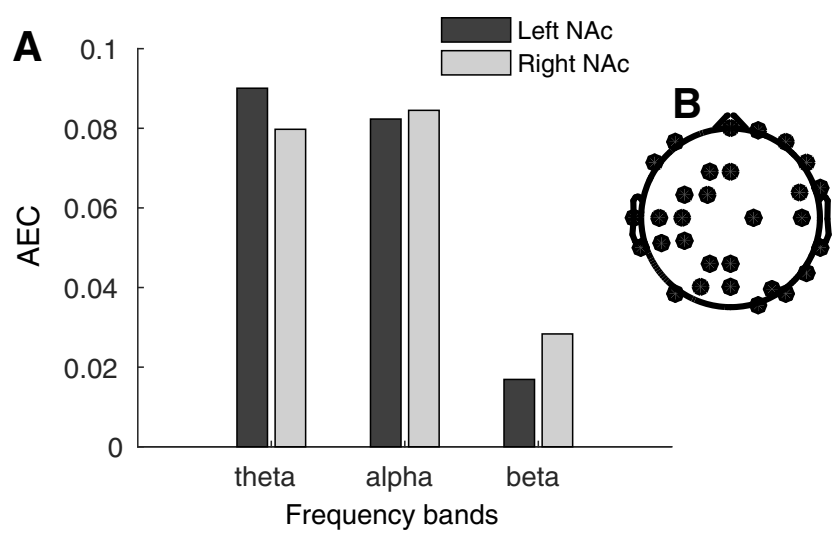

Figure 1. $\mathrm{AEC}$ in the $\theta(4-8 \mathrm{~Hz}), \alpha(8-13 \mathrm{~Hz})$, and $\beta(13-30 \mathrm{~Hz})$ frequency bands on the overall task. $\boldsymbol{A}$, The bars depict average AEC between the NAc (most ventral contact point of the DBS electrode) and 30 randomly selected surface EEG channels, for the left and right NAc separately. $\boldsymbol{B}$, Topology of the 30 randomly selected surface electrodes.

with Hamming window) in the $\theta(4-8 \mathrm{~Hz})$ and $\alpha(8-13 \mathrm{~Hz})$ frequency ranges. To check for potential relevance of $\beta$ oscillations, we looked at overall task $\beta$ connectivity. For this, we calculated amplitude envelope correlation (AEC), an amplitude-based connectivity measure that allows for some variability in frequency between signals (further description below), for the $\theta, \alpha$, and $\beta(13-30 \mathrm{~Hz}$ ) frequency bands between bilateral NAc and 30 randomly selected surface EEG channels on overall task data (per subject an average of 256 trials of all conditions combined, lasting from -500 to $2200 \mathrm{~ms}$ relative to the go signal). We then compared these frequency bands using a one-way ANOVA $\left(F_{(2,18)}=4.12\right.$, $p=0.034)$ and subsequent two-sample $t$ tests, which indicated significantly less connectivity in the $\beta$-band, compared with the $\theta$-band $\left(t_{(12)}=2.7, p=0.019\right)$ and $\alpha$-band $\left(t_{(12)}=2.97, p=0.012\right)$. Figure 1 depicts $\mathrm{AEC}$ on the overall task per frequency band, averaged over the NAc, surface EEG channels pairs, for the left and right NAc separately, as well as the topology of the selected surface electrodes. Based hereon, $\beta$-band oscillations were excluded from further analyses.

\section{Connectivity measures}

We have adopted two complementary connectivity measures that rely on different oscillatory characteristics to detect coupling between anatomically distributed sources: amplitude and phase. First, AEC detects coupling based on correlated amplitude modulations, thought to reflect the extent of synchrony of neural assemblies (Varela et al., 2001), thus independently of presence of phase coherence and differences in frequency. This method was found a suitable complementary measure to coherence for detecting longer-range, polysynaptic, cortical $\gamma$ interaction in humans (Bruns et al., 2000) and subcortical-cortical $\beta-\gamma$ coupling in LFPs recorded in cats (Bekisz and Wróbel, 1999). We calculated AEC by correlating the Hilbert envelopes of the signals. Second, directed phase transfer entropy (dPTE) estimates the direction of information flow using transfer entropy between instantaneous phase time-series. It was implemented as described in detail by Lobier et al. (2014), who showed that it quantifies directed connectivity in a model-free manner that is robust to realistic amounts of noise and linear mixing. First, timeseries were complex filtered using the Hilbert transform, then the phase angle was extracted from the complex signals using angle, which were put in a range of $0-2^{*} \pi$. The phases were binned using the number of bins defined in Scott (1992). These binned phases were compared with phases of the second signal after a predefined lag of $\sim 10 \mathrm{~ms}$. Since lag precision was restricted by sampling rate, ultimately, approximation of the 10-ms interaction lag was $11.7 \mathrm{~ms}$. Transfer entropy, which is based on the principle that, if signal $X$ influences signal $Y$, the probability density of signal Y's future conditioned on its past should differ from that conditioned on the pasts both signals $X$ and Y (Schreiber, 2000), with the probability density quantified by Shannon Entropy (Shannon, 1948). Lastly, dPTE was normalized using the marginal probability densities (i.e., within signal transfer entrophies), resulting in values ranging between 0 and 1, with $0.5-1$ indicating influence of $X$ over $Y$, $0-0.5$ indicating influence of $Y$ over $X$, and 0.5 indicating absence of preferential direction of information flow. We used MATLAB to implement dPTE. We calculated both connectivity measures over the entire trial lengths to optimize the accuracy of the low frequency phase estimates, considering their strong dependence hereon.

\section{Power spectral density (PSD)}

We calculated PSD separately for the $550 \mathrm{~ms}$ before and after the event (stop signal onset or motor response). To this end, we first applied a fast Fourier transform. Then, to calculate PSD in decibel (dB), we used this Fourier transformed data as input for the following formula: $10^{\star} \log 10(((1)$ (srate*sum(slength))) * $\left.\left.a b s(F) \wedge^{\wedge} 2\right)^{*} 2\right)$, where $F$ is the Fourier transformed data, srate is the sampling rate, and slength is the number of samples in the signal. With a frequency resolution of $1.8 \mathrm{~Hz}$, we averaged the PSD at $\sim 3.6,5.4$, and $7.2 \mathrm{~Hz}$ for the $\theta$ frequency PSD and 9, 10.8, and $12.6 \mathrm{~Hz}$ for the $\alpha$ frequency PSD. From here on out, we will refer to PSD as power.

\section{Statistical analyses}

We employed three levels of correction in this descriptive study. First, to account for the dependency across trials within subject, we applied linear mixed-effects modeling (LMM; MATLAB's fit/me) with random effects for subject. Second, to correct for the total number of channels tested and account for non-normality, we employed 10,000-iteration permutation tests with maximum and minimum $t$ distributions. This is a method generally used to control the family wise error rate in neuroimaging research, yet it is also suitable for electrophysiological data (Kilner et al., 2005). Third, we Bonferroni-corrected for the number of frequency bands, connectivity measures, and hemispheres tested. We tested the most ventral iEEG channel per hemisphere, located in the NAc, and EEG channels that previously showed connectivity with the NAc during cognitive flexibility (Horschig et al., 2015). These were channels Fp1, Fpz, Fp2, AF7, F7, F1, Fz, FCz, P1, Pz, P2, POz, PO4, O1, and O2. Since subject 6 lacked 
usable signal from the right NAc, analyses on this channel are based on six instead of seven participants.

For the connectivity analyses, the LMM included random effects for subject and a fixed effect for condition. We tested the connectivity measures separately and over the entire epoch, as opposed to pre. Per iteration of the permutation test, we first randomly shuffled the condition labels within subject before fitting the LMM. The labels were identically shuffled for $\theta$ and $\alpha$-filtered signals. Then, we took the maximum and minimum $t$ values across all channels to form the null distributions. For dPTE, the 2.5th percentile of the minimum $t$ distribution and the 97.5th percentile of the maximum $t$ distribution constituted the critical values for the lower and upper tails, respectively, consistent with two-tailed testing. Because dPTE is a directed measure, testing both tails reflects testing for both cortex-to-NAc and NAc-to-cortex communication. For AEC, the 95th percentile of the maximum $t$ distribution constituted the critical value for the upper tail, consistent with one-tailed testing. This reflect testing for coupling, but not decoupling, of signals. Ultimately, we Bonferroni-corrected for four comparisons (two frequency bands $\times$ two connectivity measures), resulting in a critical $p$ value of 0.0125 .

For the power analyses, the LMM included random effects for subject and fixed effects for condition, time, and the interaction between condition and time (pre vs post event). We shuffled the condition labels within subject and time-period (pre or post event), formed null distributions of the maximum and minimum $t$ values per iteration and performed two-tailed tests as described above. However, here, we tested the iEEG channels against their individual null distributions instead of being collapsed with the EEG channels (and corrected for this via Bonferroni correction), whereas the null distributions for the EEG channels were based on all EEG channels (identical to the connectivity analyses), thereby correcting for the number of channels tested. Ultimately, we Bonferronicorrected the iEEG channels for four comparisons (two frequency bands $\times$ two hemispheres) and the EEG channels for two comparisons (two frequency bands), resulting in critical $p$ values of 0.0125 and 0.025 , respectively.

\section{Post hoc testing}

Significant condition effects in connectivity were subjected to post hoc testing to inform about the timing of the found effect. This included calculating the relevant connectivity measure for a 500-ms sliding window with a stepsize of $23.4 \mathrm{~ms}$, resulting in 26 time windows. For stepsize, we approximated $25 \mathrm{~ms}$, yet precision was restricted by sampling rate. Solely for visualization purposes, we interpolated missing electrodes using spherical spline interpolation (EEGLAB toolbox). For visualization of the sliding window analysis, we oversampled (factor 5) and smoothed (two-point moving average, i.e., 10-point for the oversampled data) the data.

To see whether significant condition effects in connectivity were specific to the most ventrally located contact point of the DBS electrode (L/R0), targeted at the NAc, we tested whether the effect(s) could also be found on the most dorsally located DBS contact point (L/R3). As expected, when average referencing included R0, power spectra for R3 consistently showed lower power than when average referencing excluded Ro. This suggests that the signal measured at L/R0 contains considerably higher spectral power. Therefore, we referenced L/R3 against all available other contact points of the DBS electrode (L/R1/L/R2) except for L/R0. For just comparison, we repeated the 10,000-iteration permutation test, using the signals from L/R3 instead of L/R0 to calculate connectivity with the surface electrodes. Lastly, considering hemispheric lateralization of motor planning and execution (Sabate et al., 2004), we checked for lateralization of significant connectivity results by adding a main effect of side (left/right trial) and its interaction with condition to the LMM and applying this to the relevant channel pair(s).

For significant power results, we calculated the percentage of change in power over time using the following formula: $\left(10^{\wedge}(\mathrm{diff} / 10)-1\right)^{\star} 100$, where diff is the difference in grand average from pre to post event. Additionally, we tested whether $\alpha$ and $\theta$ power changes were related on a trial-by-trial basis by applying LMMs on the pre-to-post power changes with random effects for subject. We also tested whether significant power modulations were specific to the most ventrally located contact point of the DBS electrode (L/R0) or could also be found on the most dorsally located contact point (L/R3). L/R3 was tested against its own maximum $t$ distribution, resulting from a 10,000-iteration permutation. Lastly, we checked for lateralization of power modulation by adding a main effect of side (left/right trial) and its interactions with condition and time (pre or post event) to the LMM and applying this to the relevant channel pair(s).

\section{Results}

\section{Task performance}

Table 1 shows sample characteristics and behavioral performance. On average, participants showed an SSRT of $255 \mathrm{~ms}$, indicative of the time required to inhibit an already initiated motor response, they successfully inhibited their response in $52 \%$ of stop trials, and either failed to respond or responded incorrectly in $2 \%$ of go trials. Mean RTs on correct go and failed inhibition trials were 679 and $594 \mathrm{~ms}$, respectively. The former seems considerably longer and somewhat more variable than generally reported for both healthy participants and OCD patients (Penadés et al., 2007; Boisseau et al., 2012). However, whereas OCD patients usually show longer SSRTs than controls (Lipszyc and Schachar, 2010), current SSRT lies within the ranges reported for both controls and patients; seemingly somewhere in between their means, yet the literature shows considerable variability. Notably, the depressed participants showed the most omissions $(13 \%$ and $2 \%$ ) on go trials.

\section{Connectivity between NAc and the cortex}

When comparing connectivity during the $-550-550 \mathrm{~ms}$ relative to stop signal onset between successful and failed inhibition trials, we found that inhibition success was associated with more negative dPTE between the 

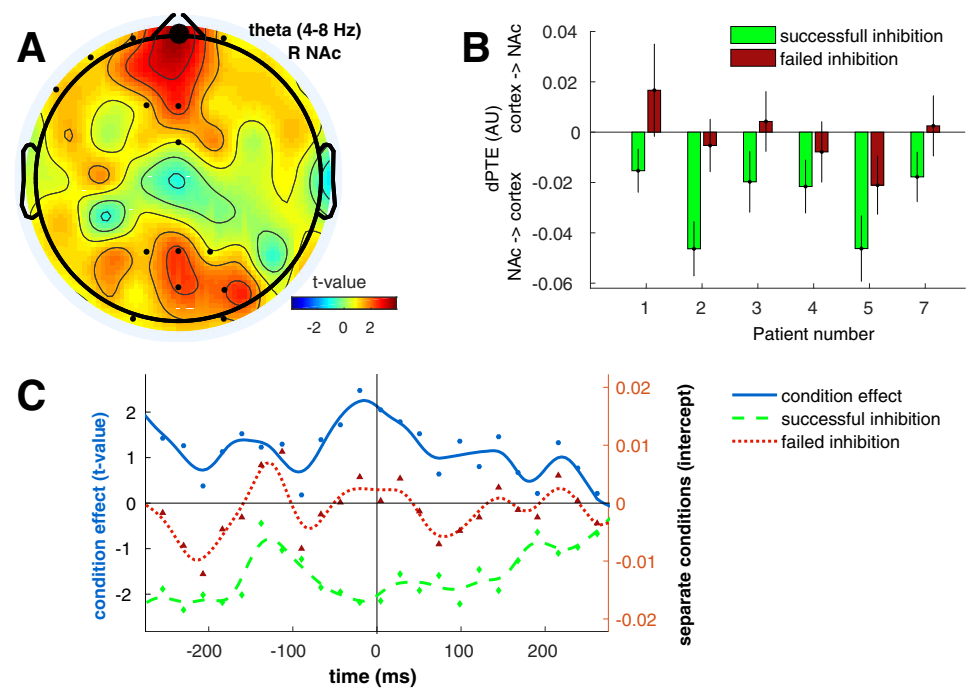

Figure 2. Effect of inhibition success on dPTE between right NAc and scalp electrodes. $\boldsymbol{A}$, dPTE between right NAc and EEG electrode Fpz (large dot) showed a condition effect in the $\theta$-band on successful versus failed inhibition trials $(-550-550 \mathrm{~ms}$ relative to stop stimulus onset). LMM $t$ values are plotted with small dots indicating tested channels. $\boldsymbol{B}$, Mean dPTE (arbitrary units, centered) for conditions and patients separately. Positive and negative values indicate cortex $\rightarrow N A c$ and NAc $\rightarrow$ cortex information flow, respectively. Error bars indicate SEM. C, Post hoc sliding window analysis showed the effect was highest just before stop stimulus onset (time $=0$ ). Condition effect $t$ values (solid line) were smoothed and plotted on the left $y$-axis, whereas the right $y$-axis reflects centered smoothed dPTE intercepts (dashed lines) for the separate conditions, with negative values again indicating effective connectivity from the NAc toward the cortex and vice versa. Since dPTE was calculated for a sliding window, with each dot representing $500 \mathrm{~ms}$, the approximately $-290-290 \mathrm{~ms}$ shown on the $x$-axis represents the entire -550 - to 550 -ms trial length.

right NAc and Fpz in the $\theta$-band (Fig. 2A; Table 2). This effect was stable across subjects (Fig. 2B). Post hoc sliding window analysis revealed that this effect, which reflects information flow from the NAc to Fpz, was already present before stop stimulus onset (Fig. 2C). This effect was not different for left versus right trials $\left(t_{(462)}=0.73, p=0.466\right)$, nor did it show an interaction effect between condition and side $\left(t_{(462)}=0.28, p=0.779\right)$. Furthermore, $\theta$-band dPTE between R3, the most dorsally located DBS contact point, and $\mathrm{Fpz}$ did not show a significant condition effect $\left(t_{(464)}=2.52, p=0.073, p\right.$-Bonferroni-corrected $\left.=0.291\right)$, suggesting that the effect is local to $R 0$, the most ventrally located DBS contact points, targeted at the NAc. No effects were found for AEC or connectivity in the $\alpha$-band.

When comparing connectivity during the $-550-550 \mathrm{~ms}$ relative to motor response between failed inhibition and correct go trials, we did not find any effects after Bonferroni correction. Before correction for four comparisons, we saw more positive AEC between the right NAc and 01 in the $\theta$-band $\left(t_{(464)}=2.76, p=0.0442\right)$ and more positive dPTE between the left NAc and P1 in the $\alpha$-band $\left(t_{(519)}=3.21\right.$, $p=0.022$; Table 3 ) on failed inhibition compared with correct go trials.

\section{Power modulation in the NAc and the cortex}

When comparing power between successful and failed inhibition trials and changes between the 550 -ms pre versus post stop signal onset, we did not find significant effects for condition or the interaction between time and condition after Bonferroni correction. Neither $\theta$ nor $\alpha$ power modulation around stop signal onset seems to underlie inhibition success.
When comparing power between failed inhibition and correct go trials and changes between the 550-ms pre versus post motor response, we found a greater $\theta$ power increase after response on failed inhibition trials compared with correct go trials in the left NAc and at electrode FCz (Fig. 3A,B; Table 4). This was accompanied by a greater decrease in $\alpha$ power at electrode PO4 (Fig. 3C). These changes in power from pre-to-post response on correct go and failed inhibition trials, respectively, were $5 \%$ and $43 \%$ for the left NAc, $10 \%$ and $75 \%$ for $\mathrm{FCz}$, and $1 \%$ and $29 \%$ for PO4. Figure $3 D-F$ shows the variability of these effects over subjects. We found no lateralization of $\theta$ power modulation in the left NAc (side: $t_{(872)}=0.44$, $p=0.657$; side $\times$ condition $\times$ time: $t=1.73, p=0.083$ ) or FCz (side: $t=0.11, p=0.911$; side $\times$ condition $\times$ time: $t=0.13, p=0.894$ ), nor of $\alpha$ power modulation on PO4 (side: $t=0.43, p=0.669$; side $\times$ condition $\times$ time: $t=0.40$, $p=0.689$ ). Before Bonferroni correction for four comparisons, we additionally found an effect of condition on $\alpha$ power in the left NAc, showing $13 \%$ more $\alpha$ power on failed inhibition compared with correct go trials $\left(t_{(876)}=\right.$ $1.87, p=0.0284)$. Post hoc LMMs indicated no significant linear relationship between the changes in $\alpha$ power at PO4 and $\theta$ power in the left NAc $\left(t_{(218)}=-1.6, p=0.1138\right)$ or at FCz $\left(t_{(218)}=-1.47, p=0.1430\right)$. Specificity analysis showed a significant, yet somewhat smaller, interaction effect on $\theta$ power at the most dorsally located contact point of the left DBS electrode $(t=3.26, p=0.0007, p$ Bonferroni-corrected $=0.0028$ ). The effect showed a similar pattern to that found on the most ventrally located contact point, with pre-to-post response power changes of $-1 \%$ and $41 \%$ on correct go and failed inhibition trials, respectively. 


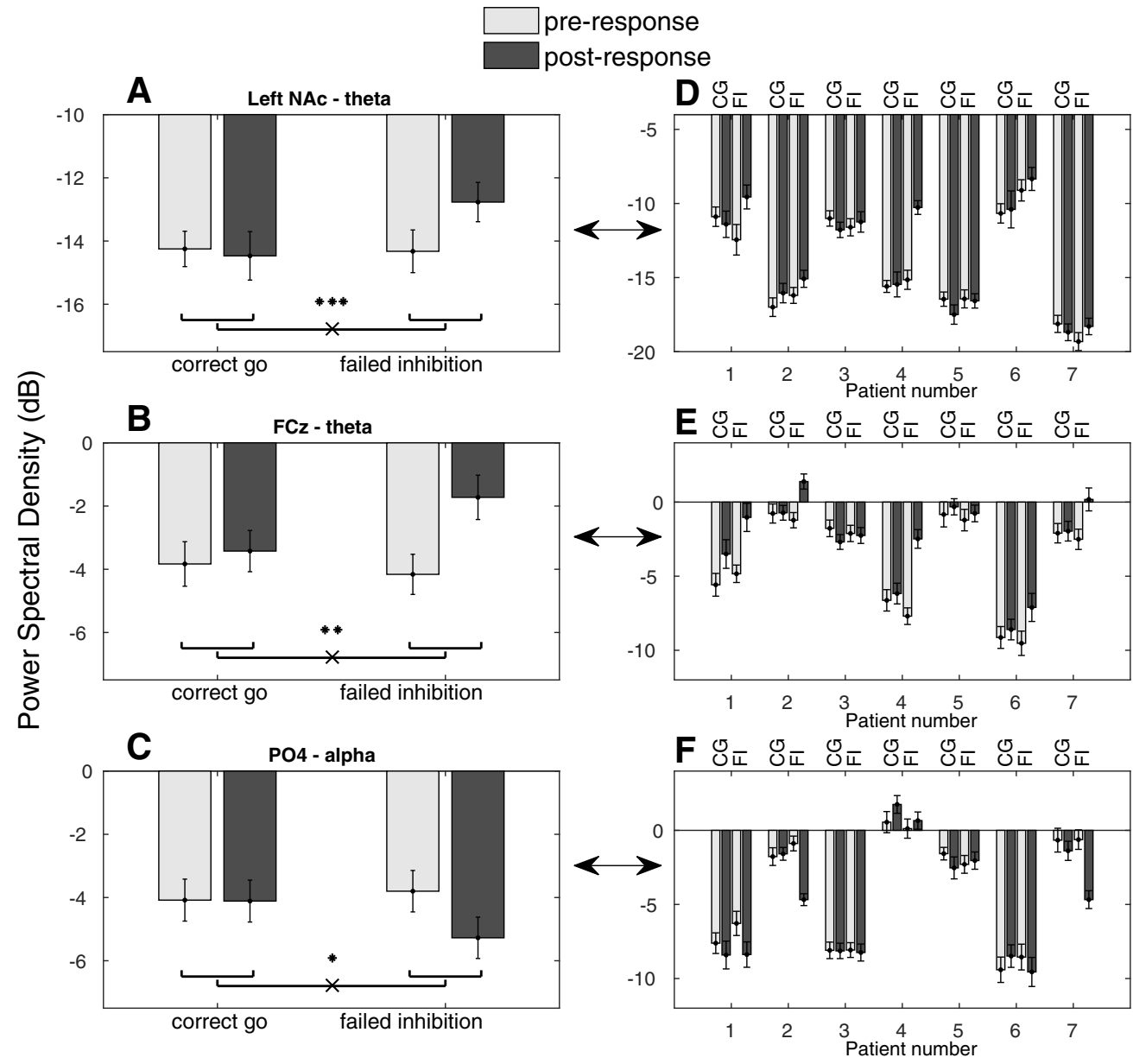

Figure 3. PSD modulation following response on failed inhibition versus correct go trials. Power is expressed in decibel (dB). Error bars indicate SEM. Greater $\theta$ power increase following motor response on failed inhibition compared with correct go trials $(\boldsymbol{A})$ in the left NAc and $(\boldsymbol{B})$ on electrode FCz. $\boldsymbol{C}$, Greater $\alpha$ power decrease following motor response on failed inhibition compared with correct go trials on electrode PO4. $\boldsymbol{D}-\boldsymbol{F}$, Power modulation from plots $\boldsymbol{A}-\boldsymbol{C}$, respectively, visualized for patients separately. CG and FI refer to correct go and failed inhibition conditions, respectively. ${ }^{* * *} p<0.0005$ before and 0.002 after Bonferroni correction, ${ }^{* *} p<0.001$ before and 0.005 after Bonferroni correction, ${ }^{*} p<0.05$.

\section{Discussion}

We found that inhibition success, as opposed to failure, was associated with increased information flow from right NAc to medial frontal cortex through phase coupling of $\theta$ oscillations, present already before stop signal onset. We additionally found that $\theta$ power increased following motor response on failed inhibition compared with correct go trials in both the left NAc and medial frontal cortex, whereas parieto-occipital cortex showed an $\alpha$ power decrease.

To our knowledge, this is the first report to show involvement of the NAc and its communication with frontal cortex in adaptive behavior pertaining motor control. Lack of significant findings for AEC or in the $\alpha$-band suggests $\theta$

Table 2: Channel availability

\begin{tabular}{llllc}
\hline & $\begin{array}{l}\text { Intracranial contact } \\
\text { points rejected }\end{array}$ & $\begin{array}{l}\text { EEG channels missing } \\
\text { from selection }\end{array}$ & $\begin{array}{l}\text { Number of EEG } \\
\text { channels rejected }\end{array}$ & $\begin{array}{l}\text { Number of EEG channels } \\
\text { not recorded }\end{array}$ \\
\hline Patient 1 & R1 & AF7 & 3 , including AF7 & 9 \\
Patient 2 & R2 & AF7 & 1, including AF7 & 9 \\
Patient 3 & R1 & O1, Oz, O2 & 6 , including O1, Oz, O2 & 8 \\
Patient 4 & R2 & Fp2 & 6 & 8 \\
Patient 5 & R1 & & 0 & 6 \\
Patient 6 & R0, R1 & AF7 & 10, including AF7 & 10 \\
Patient 7 & & & 4 & 8
\end{tabular}

${ }^{\mathrm{a}} \mathrm{R}=$ right hemisphere, $0=$ most ventral contact point, located in the NAc, $1-2=$ contact points one and two places, respectively, more dorsal from the most ventral contact point/NAc. 
Table 3: Condition effects in connectivity between NAc and the cortex

\begin{tabular}{|c|c|c|c|c|c|c|c|}
\hline Time-locking: conditions & $\begin{array}{l}\text { Connectivity } \\
\text { measure }\end{array}$ & $\begin{array}{l}\text { Frequency } \\
\text { band }\end{array}$ & $\begin{array}{l}\text { NAc } \\
\text { hemisphere }\end{array}$ & EEG & $t$ value & $\begin{array}{l}p \text { value } \\
\text { before } \\
\text { Bonferroni } \\
\text { correction }\end{array}$ & $\begin{array}{l}p \text { value } \\
\text { after } \\
\text { Bonferroni } \\
\text { correction }\end{array}$ \\
\hline \multirow[t]{2}{*}{ Response: failed inhibition vs correct go* } & AEC & $\theta$ & $\mathrm{R}$ & 01 & 2.76 & 0.0442 & 0.1768 \\
\hline & dPTE & $\alpha$ & $\mathrm{L}$ & P1 & 3.21 & 0.0220 & 0.0880 \\
\hline
\end{tabular}

* Solely significant before Bonferroni correction.

phase specificity of NAc-frontal cortex communication underlying inhibition success. However, some trends suggested the possibility of $\theta$ amplitude coupling between right NAc and (left) occipital cortex and/or $\alpha$ phase coupling between the left NAc and (left) parietal cortex to distinguish between correct going and failed inhibition.

The observed connectivity is consistent with the finding of NAc-to-frontal cortex $\theta$-band granger causality during anticipation of a visual stimulus during a task of cognitive flexibility (Horschig et al., 2015). We likewise found that communication was already present before, and sustained around, stimulus presentation. Computational models suggest that the ventral striatum might actively gate sensory information based on task demands maintained in frontal regions of cognitive control (Frank et al., 2001). This idea was previously substantiated by showing that the NAc modulated fronto-parietal coherence in the $\alpha$-band, which is in line with a nonlinear dynamic causal modeling $\mathrm{fMRI}$ study showing that shifts in attention relied on the ventral striatopallidum to modulate connectivity between stimulus-specific visual association areas and the prefrontal cortex (van Schouwenburg et al., 2010). Therefore, the currently found prestimulus phase coupling between NAc and frontal cortex might likewise reflect facilitation of task-relevant information. Considering the visual SST, this information likely originates from visual cortex and flows via the globus pallidus, subthalamic nucleus, and thalamus to frontal cortex (Haber et al., 1995; Haber and Knutson, 2010). In line with this view, we found a trend toward $\theta$ amplitude coupling between right NAc and electrode 01 . These findings extend the well-established role of the NAc in reward processing and reinforcement learning (Cohen et al., 2009a, 2012; Lega et al., 2011; Patel et al., 2012) to the context of adaptive behavior in tasks of both cognitive and behavioral flexibility.

However, we did not observe the poststimulus increase in information flow from the NAc to the cortex that was previously found (Horschig et al., 2015). This might be explained by task differences, since stimulus presentation in the former task required redirection of attention and a subsequent button press, whereas it required inhibition of a button press in the present task. In addition, it previously was unclear whether the effects relied on phase or amplitude locking, because granger causality depends on both such signal components (Lobier et al., 2014). We now extend those findings by showing that the information flow from the NAc to the cortex depends on $\theta$ phase synchronization, rather than amplitude coupling. In contrast to other intracranial studies that found connectivity from the cortex to the NAc during attentional switching and reward anticipation, we did not find information flow from the cortex to the NAc, being indicative of cognitive control, to underlie inhibition success (Cohen et al., 2012; Horschig et al., 2015).

We additionally found an increase in midfrontal and left NAc $\theta$ power as well as a decrease in parieto-occipital $\alpha$ power after response on failed inhibition compared with correct go trials. $\theta$ Power increases in both NAc and medial frontal cortex have previously been found following, especially negative, feedback on a variety of tasks (Cohen et al., 2007, 2009b; Münte et al., 2008; Nurislamova et al., 2019). The well-established feedback or error-related negativity, evoked by erroneous (motor) responses, has been found to arise from a combination of a power increase and partial phase synchronization of $\theta$ oscillations (Luu et al., 2004; Trujillo and Allen, 2007). These signals have been traced back to the anterior cingulate cortex and/or pre-SMA, which are involved in error and conflict processing and subsequent behavioral adjustment (Garavan et al., 2002; Luu et al., 2004; lannaccone et al., 2015). Interestingly, it has been postulated that, whereas high $\theta$ might reflect the conflict monitoring process itself, low $\theta$ underlies the more general process of interregional communication and thus relays the error to other areas of cognitive control (Huster et al., 2013). Current power increases were more pronounced for lower $\theta$ frequencies, especially so for electrode $\mathrm{FCz}$, pointing to engagement of cognitive control after failed inhibition. Performance monitoring-related $\theta$ power increases often co-occur with increased $\theta$ phase synchronization between medial

Table 4: PSD modulation following motor response on failed inhibition versus correct go trials

\begin{tabular}{|c|c|c|c|c|c|}
\hline Frequency band & Effect & Channel & $t$ value & $\begin{array}{l}p \text { value before } \\
\text { Bonferroni correction }\end{array}$ & $\begin{array}{l}p \text { value after } \\
\text { Bonferroni correction }\end{array}$ \\
\hline \multirow[t]{2}{*}{$\theta$} & Condition $\times$ time & NAc L & 3.29 & 0.0004 & 0.0016 \\
\hline & Condition $\times$ time & $\mathrm{FCz}$ & 3.94 & 0.0006 & 0.0012 \\
\hline$\alpha$ & Condition $\times$ time & $\mathrm{PO} 4$ & -3.11 & 0.0110 & 0.0220 \\
\hline
\end{tabular}

\footnotetext{
* Solely significant before Bonferroni correction.
} 
frontal and parietal cortex (Nurislamova et al., 2019), which was previously found to be modulated by the NAC during attentional switching (Horschig et al., 2015). Although we found a concurrent decrease in posterior $\alpha$ power, something previously found to accompany midfrontal $\theta$ increases following failed inhibition on a Go/ NoGo task (Mazaheri et al., 2009), these $\theta$ and $\alpha$ power modulations were not significantly related on a trial-bytrial basis. However, with $p=0.11$ and $p=0.14$ for those relationships, this might have resulted from our limited sample size. $\alpha$ Power increases are thought to decrease local neural processing capacity, thereby inhibiting a region's activity (Jensen and Mazaheri, 2010). Therefore, our $\alpha$ power decrease might reflect release of inhibition on the posterior parietal regions involved in action planning and decision-making, likely resulting from top-down influence of the frontal control system (Andersen and Cui, 2009).

In conclusion, our results supplement current knowledge about cortical involvement in performance monitoring by implicating NAc $\theta$ power modulation in the engagement of cognitive control after inhibition failure, possibly for subsequent adjustment of decision-making parameters to prevent additional errors. This extends the previous finding that subthalamic nucleus $\theta$ power and coherence with frontal cortex are likewise modulated during the SST (Alegre et al., 2013). Similar to the $\theta$ power increases we found in the $\mathrm{NAc}$ and frontal cortex and relate to the well-established error-related negativity, the subthalamic nucleus showed inhibition failure-related increases in $\theta$ power and coherence with frontal cortex. If and how communications between these subcortical structures underlies feedback processing remains unanswered, yet these findings point to a role of $\theta$ oscillations herein. Additionally, we found inhibition success-related $\theta$ connectivity between the NAc and frontal cortex that was absent in the subthalamic nucleus. Although the inhibition success-related connectivity was specific to the most ventrally located contact point of the right DBS electrode, targeted at the NAc, we found the postresponse $\theta$ power increase also on the most dorsally located contact point of the left DBS electrode. Although all targeted at the NAc, slight differences in DBS electrode location between patients could explain this non-specificity, considering that the medially located contact points were used as reference. Alternatively, it could be that the power change is not restricted to the gray matter of the NAc. Lack of significant lateralization of our main effects substantiates their interpretation as higher-order regulatory rather than primary motor processes (Sabate et al., 2004).

Notwithstanding the unique dataset, it comes with some limitations. First of all, although we allowed for random effects for subject in our models, the sample size of seven subjects limits statistical power and generalizability of the results. To account for this and limit (unnecessary) multiple comparison correction, we tested a selection of EEG channels based on previous research (Horschig et al., 2015), yet thereby limiting exploration of potentially unexpected findings. Also, we were unable to measure the impedances of the DBS electrode contact points, informative of signal quality, since doing so could potentially induce non-therapeutic stimulation. Furthermore, we must keep in mind that our results might represent pathologic brain functioning, since we used a severely affected psychiatric sample and lacked a control group. Especially so since NAc-DBS is thought to exert its therapeutic effects through targeting NA-cortical connectivity (Figee et al., 2013; Smolders et al., 2013). Also, MDD patients show aberrant error-related negativity (Tucker et al., 2003; Holmes and Pizzagalli, 2008), which has been linked to midline frontal $\theta$ oscillations during action regulation (Luu and Tucker, 2001). However, cortical feedback-related negativity has been found not to differ between controls and DBS-implanted OCD and Tourette's syndrome patients (Schüller et al., 2015) and we found stable and significant results in a sample that included various disorders, albeit mainly disorders of compulsivity. Moreover, participants were not taking SSRIs at the time of data collection, yet postsurgical analgesics could have affected brain functioning.

We found condition-specific phase-synchronization and power modulation for separate time periods of task performance, yet associations between oscillatory phase and power, a phenomenon called cross-frequency coupling, have additionally been reported. Coupling of $\gamma$ power to $\alpha$ phase in the NAc was found during reward processing (Cohen et al., 2009a), decreased before strategic switching (Cohen et al., 2009b), and differentiated between positive and negative feedback (Lega et al., 2011). Moreover, NAc $\gamma-\theta$ coupling varied with cognitive control during a motor learning task (Dürschmid, 2013). To gain more insight into the interplay between subcortical and cortical local cross-frequency coupling and phase synchronization between distant regions, such associations should be tested directly using datasets such as ours. Additionally, since increased $\theta$ phase synchronization between bilateral NAc has been linked to behavioral adjustment following losses (Cohen et al., 2009b), interNAc connectivity might also be relevant for SST performance. Lastly, the possibility of functional hemispheric differentiation of the NAc warrant further investigation, considering we found right lateralized inhibition successrelated connectivity changes and left lateralized performance monitoring-related power changes.

In sum, because of our unique dataset of concurrent striatal and EEG recordings, we were able to show involvement of prestimulus NAc-to-medial frontal cortex $\theta$ phase synchronization in successful response inhibition and both cortical and NAc power modulation in the $\theta$-band and $\alpha$-band in performance monitoring on the SST. These results corroborate earlier findings that $\theta$ oscillations are crucial for cortical-subcortical communication during cognitive processing and involvement of the NAc in adaptive behavior. However, still plenty remains to be learned about both the specificity and the extent of interplay of different features of oscillatory activity, including cross-frequency coupling and the relationship between NAc-cortical communication and cortical interactions. 


\section{References}

Alegre M, Lopez-Azcarate J, Obeso I, Wilkinson L, Rodriguez-Oroz MC, Valencia M, Garcia-Garcia D, Guridi J, Artieda J, Jahanshahi M, Obeso JA (2013) The subthalamic nucleus is involved in successful inhibition in the stop-signal task: a local field potential study in Parkinson's disease. Exp Neurol 239:1-12.

Alexander GE, DeLong MR, Strick PL (1986) Parallel organization of functionally segregated circuits linking basal ganglia and cortex. Annu Rev Neurosci 9:357-381.

Andersen RA, Cui H (2009) Intention, action planning, and decision making in parietal-frontal circuits. Neuron 63:568-583.

Band GPH, van der Molen MW, Logan GD (2003) Horse-race model simulations of the stop-signal procedure. Acta Psychol (Amst) 112:105-142.

Bekisz M, Wróbel A (1999) Coupling of beta and gamma activity in cortico-thalamic systems of cats attending to visual stimuli. Neuroreport 10:3589-3594.

Bissett PG, Logan GD (2011) Balancing cognitive demands: control adjustments in the stop-signal paradigm. J Exp Psychol Learn Mem Cogn 37:392-404.

Boisseau CL, Thompson-Brenner H, Caldwell-Harris C, Pratt E, Farchione T, Barlow DH (2012) Behavioral and cognitive impulsivity in obsessive-compulsive disorder and eating disorders. Psychiatry Res 200:1062-1066.

Bruns A, Eckhorn R, Jokeit H, Ebner A (2000) Amplitude envelope correlation detects coupling among incoherent brain signals. Neuroreport 11:1509-1514.

Cohen MX, Elger CE, Ranganath C (2007) Reward expectation modulates feedback-related negativity and EEG spectra. Neuroimage 35:968-978

Cohen MX, Axmacher N, Lenartz D, Elger CE, Sturm V, Schlaepfer TE (2009a) Good vibrations: cross-frequency coupling in the human nucleus accumbens during reward processing. $\mathrm{J}$ Cogn Neurosci 21:875-889.

Cohen MX, Axmacher N, Lenartz D, Elger CE, Sturm V, Schlaepfer TE (2009b) Nuclei accumbens phase synchrony predicts decisionmaking reversals following negative feedback. J Neurosci 29:7591-7598.

Cohen MX, Bour L, Mantione M, Figee M, Vink M, Tijssen MA, van Rootselaar AF, van den Munckhof P, Schuurman PR, Denys D (2012) Top-down-directed synchrony from medial frontal cortex to nucleus accumbens during reward anticipation. Hum Brain Mapp 33:246-252.

Delorme A, Makeig S (2004) EEGLAB: an open source toolbox for analysis of single-trial EEG dynamics including independent component analysis. J Neurosci Methods 134:9-21.

Dürschmid S (2013) Phase-amplitude cross-frequency coupling in the human nucleus accumbens tracks action monitoring during cognitive control. Front Hum Neurosci 7:1-17.

Figee M, Luigjes J, Smolders R, Valencia-Alfonso CE, van Wingen G, de Kwaasteniet B, Mantione M, Ooms P, de Koning P, Vulink N, Levar $N$, Droge $L$, van den Munckhof $P$, Schuurman PR, Nederveen A, van den Brink W, Mazaheri A, Vink M, Denys D (2013) Deep brain stimulation restores frontostriatal network activity in obsessive-compulsive disorder. Nat Neurosci 16:386-387.

Floresco SB, Ghods-Sharifi S, Vexelman C, Magyar O (2006) Dissociable roles for the nucleus accumbens core and shell in regulating set shifting. J Neurosci 26:2449-2457.

Frank MJ, Loughry B, O’Reilly RC (2001) Interactions between frontal cortex and basal ganglia in working memory: a computational model. Cogn Affect Behav Neurosci 1:137-160.

Garavan H, Ross TJ, Murphy K, Roche RA, Stein EA (2002) Dissociable executive functions in the dynamic control of behavior: inhibition, error detection, and correction. Neuroimage 17:18201829.

Gomez-Herrero G, Clercq W, Anwar H, Kara O, Egiazarian K, Huffel S, Paesschen W (2006) Automatic removal of ocular artifacts in the EEG without an EOG reference channel. Proceedings of the 7 th
Nordic Signal Processing Symposium - NORSIG Reykjavik, Iceland, 7-9 June 2006, 2006, pp 130-133.

Haber SN, Knutson B (2010) The reward circuit: linking primate anatomy and human imaging. Neuropsychopharmacology 35:4-26.

Haber SN, Kunishio K, Mizobuchi M, Lynd-Balta E (1995) The orbital and medial prefrontal circuit through the primate basal ganglia. $J$ Neurosci 15:4851-4867.

Holmes AJ, Pizzagalli DA (2008) Spatiotemporal dynamics of error processing dysfunctions in major depressive disorder. Arch Gen Psychiatry 65:179-188.

Horschig JM, Smolders R, Bonnefond M, Schoffelen JM, van den Munckhof P, Schuurman PR, Cools R, Denys D, Jensen O (2015) Directed communication between nucleus accumbens and neocortex in humans is differentially supported by synchronization in the theta and alpha band. PLoS One 10:e0138685.

Huster RJ, Enriquez-Geppert S, Lavallee CF, Falkenstein M, Herrmann CS (2013) Electroencephalography of response inhibition tasks: functional networks and cognitive contributions. Int J Psychophysiol 87:217-233.

lannaccone R, Hauser TU, Staempfli P, Walitza S, Brandeis D, Brem $S$ (2015) Conflict monitoring and error processing: new insights from simultaneous EEG-fMRI. Neuroimage 105:395-407.

Jensen O, Mazaheri A (2010) Shaping functional architecture by oscillatory alpha activity: gating by inhibition. Front Hum Neurosci 4:186.

Kilner JM, Kiebel SJ, Friston KJ (2005) Applications of random field theory to electrophysiology. Neurosci Lett 374:174-178.

Lega BC, Kahana MJ, Jaggi J, Baltuch GH, Zaghloul K (2011) Neuronal and oscillatory activity during reward processing in the human ventral striatum. Neuroreport 22:795-800.

Lipszyc J, Schachar R (2010) Inhibitory control and psychopathology: a meta-analysis of studies using the stop signal task. J Int Neuropsychol Soc 16:1064-1076.

Lobier M, Siebenhühner F, Palva S, Palva JM (2014) Phase transfer entropy: a novel phase-based measure for directed connectivity in networks coupled by oscillatory interactions. Neuroimage 85:853872.

Logan GD, Cowan WB (1984) On the ability to inhibit thought and action: a theory of an act of control. Psychol Rev 91:295-327.

Luu P, Tucker DM (2001) Regulating action: alternating activation of midline frontal and motor cortical networks. Clin Neurophysiol 112:1295-1306.

Luu P, Tucker DM, Makeig S (2004) Frontal midline theta and the error-related negativity: neurophysiological mechanisms of action regulation. Clin Neurophysiol 115:1821-1835.

Mazaheri A, Nieuwenhuis IL, van Dijk H, Jensen O (2009) Prestimulus alpha and mu activity predicts failure to inhibit motor responses. Hum Brain Mapp 30:1791-1800.

Miller EK, Cohen JD (2001) An integrative theory of prefrontal cortex function. Annu Rev Neurosci 24:167-202.

Mogenson GJ, Jones DL, Yim CY (1980) From motivation to action: functional interface between the limbic system and the motor system. Prog Neurobiol 14:69-97.

Münte TF, Heldmann M, Hinrichs H, Marco-Pallares J, Krämer UM, Sturm V, Heinze HJ (2008) Contribution of subcortical structures to cognition assessed with invasive electrophysiology in humans. Front Neurosci 2:72-78.

Nurislamova YM, Novikov NA, Zhozhikashvili NA, Chernyshev BV (2019) Enhanced theta-band coherence between midfrontal and posterior parietal areas reflects post-feedback adjustments in the state of outcome uncertainty. Front Integr Neurosci 13:14.

Patel SR, Sheth SA, Mian MK, Gale JT, Greenberg BD, Dougherty DD, Eskandar EN (2012) Single-neuron responses in the human nucleus accumbens during a financial decision-making task. $\mathrm{J}$ Neurosci 32:7311-7315.

Penadés R, Catalán R, Rubia K, Andrés S, Salamero M, Gastó C (2007) Impaired response inhibition in obsessive compulsive disorder. Eur Psychiatry 22:404-410.

Ray NJ, Brittain JS, Holland P, Joundi RA, Stein JF, Aziz TZ, Jenkinson N (2012) The role of the subthalamic nucleus in 
response inhibition: evidence from local field potential recordings in the human subthalamic nucleus. Neuroimage 60:271-278.

Sabate M, Gonzalez B, Rodriguez M (2004) Brain lateralization of motor imagery: motor planning asymmetry as a cause of movement lateralization. Neuropsychologia 42:1041-1049.

Schreiber T (2000) Measuring information transfer. Phys Rev Lett 85:461-464.

Schüller T, Gruendler TO, Jocham G, Klein TA, Timmermann L, Visser-Vandewalle V, Kuhn J, Ullsperger M (2015) Rapid feedback processing in human nucleus accumbens and motor thalamus. Neuropsychologia 70:246-254.

Scott DW (1992) Multivariate density estimation. New York: Wiley, Inc.

Shannon CE (1948) A mathematical theory of communication. Bell Syst Tech J 27:379-323.

Smolders R, Mazaheri A, van Wingen G, Figee M, de Koning PP, Denys D (2013) Deep brain stimulation targeted at the nucleus accumbens decreases the potential for pathologic network communication. Biol Psychiatry 74:e27-e28.

Trujillo LT, Allen JJ (2007) Theta EEG dynamics of the error-related negativity. Clin Neurophysiol 118:645-668.
Tucker DM, Luu P, Frishkoff G, Quiring J, Poulsen C (2003) Frontolimbic response to negative feedback in clinical depression. J Abnorm Psychol 112:667-678.

van den Munckhof $P$, Bosch DA, Mantione MH, Figee M, Denys DA, Schuurman PR (2013) Active stimulation site of nucleus accumbens deep brain stimulation in obsessive-compulsive disorder is localized in the ventral internal capsule. Acta Neurochir Suppl 117:53-59.

van Schouwenburg MR, den Ouden HE, Cools R (2010) The human basal ganglia modulate frontal-posterior connectivity during attention shifting. J Neurosci 30:9910-9918.

Varela F, Lachaux J, Rodriguez E, Martinerie J (2001) The brainweb: phase synchronization and large-scale integration. Nat Rev Neurosci 2:229-239.

Verbruggen F, Logan GD (2009) Models of response inhibition in the stop-signal and stop-change paradigms. Neurosci Biobehav Rev 33:647-661.

Yawata S, Yamaguchi T, Danjo T, Hikida T, Nakanishi S (2012) Pathway-specific control of reward learning and its flexibility via selective dopamine receptors in the nucleus accumbens. Proc Natl Acad Sci USA 109:12764-12769. 